\title{
EL USO DE PESTICIDAS EN LA AGRICULTURA Y SU DESORDEN AMBIENTAL
}

The Pesticides Use in Agriculture and Their Environmental Disorder

Marco Antonio Ramírez Campos ${ }^{1, a, b .}$

${ }^{1}$ Universidad Nacional San Cristóbal de Huamanga. Ayacucho, Perú.
${ }^{a}$ Ingeniero agrónomo. ${ }^{b}$ Especialista en sanidad vegetal.

\section{RESUMEN}

El presente trabajo está basado en la recopilación de información de primer y segundo orden sobre la base teórica y los reportes de casos trascendentales, cuyo objetivo es Informar y discutir los aspectos más relevantes respecto al uso indiscriminado de pesticidas y su impacto inminente por contaminación de los ecosistemas naturales y la salud. En función a ello, los resultados se ponen en evidencia el uso de los pesticidas debido a la necesidad de contrarrestar el efecto negativo de las plagas, enfermedades y malezas sobre el rendimiento de los cultivos. No obstante, los efectos ambientales generados son promovidos por la intensificación y extensión de la agricultura como base de la alimentación en la población mundial. Además, existen muchos reportes de casos por contaminación de residuos tóxicos en las diferentes regiones del mundo que en función a ello, se establecen un conjunto de medidas de acción como propuestas concretas frente a una realidad critica que en muchos de los casos deben ser tomados en cuenta.

Palabras clave: Pesticidas, impacto, contaminación, ecosistema, población mundial.

\section{SUMMARY}

The present work is based on the collection of information of first and second order on the theoretical basis and the reports of transcendental cases, whose objective is to inform and discuss the most relevant aspects regarding the indiscriminate use of pesticides and their imminent impact due to contamination of the natural ecosystems and health. Based on this, the results show the use of pesticides due to the need to counteract the negative effect of pests, diseases and weeds on crop yields. However, the environmental effects generated are promoted by the intensification and extension of agriculture as the basis of food in the world population. In addition, there are many reports of cases of contamination of toxic waste in different regions of the world that based on this, a set of action measures are established as concrete proposals against a critical reality that in many cases must be taken in account.

Keywords: Pesticides, impact, pollution, ecosystem, world population.

\section{INTRODUCCION}

Los pesticidas son sustancias complejas de origen químico, muy necesarias en la solución de los problemas que presentan comúnmente los cultivos agrícolas, sin embargo, pueden ocasionar un conjunto de riesgos mayores a sus beneficios si no son utilizados adecuadamente.

Según las estadísticas de la FAO, dos tercios de la Humanidad se encuentran subalimentados y como consecuencia, el aumento de la producción agrícola parte de una necesidad por ampliar la frontera agrícola y el rendimiento de los cultivos (1). A nivel mundial, la producción de plaguicidas orgánicos sintéticos se incrementó desde los inicios del siglo XX como parte del desarrollo de la industria petrolera. En este caso, los plaguicidas son parte del espectro más amplio de productos químicos industriales utilizados en la sociedad moderna por las grandes compañías del mundo como forma de recurso económico. Según la base de datos de la American Chemical Society, en 1993 se identificaron más de 13 millones de productos 
químicos, a los que se suman cada año unos 500,000 nuevos compuestos (2), aumentando la carga de sustancias contaminantes en la atmósfera, suelos y agua (3), además de la desaparición de especies silvestres por efecto directo de la expansión hacia nuevas zonas de cultivo e intoxicación por residuos químicos.

\section{METODOS}

La elaboración del trabajo se realizó en base a la recopilación de textos y escritos con información de primer y segundo orden según el criterio de funcionalidad. Posteriormente se realizó un análisis y organización estructural sobre la base teórica y reportes de casos resaltantes.

\section{HISTORIA Y DESARROLLO DE LOS PESTICIDAS}

Este hecho es fundamental para entender cómo y porque los plaguicidas han representado una amenaza para el medio ambiente y porque disminuye en los países desarrollados mientras que continúa en otros (2). En la historia se han reportado catástrofes que han destruido la economía de muchos países. Es así, que en 1845 y 1846, la hambruna irlandesa fue ocasionada por la enfermedad denominada tizón tardío de la papa mientras que la hambruna bengalí de 1943 se debió a la enfermedad de la mancha café del arroz (4). En el año 2012 todas las zonas cafetaleras del mundo fueron afectadas por la roya amarilla, ocasionando grandes pérdidas en los cultivos de café. Por otro lado, las plagas también son un problema similar pero ocasionados por insectos que parasitan el ganado, destruyen la madera, se alimentan de las plantas destinadas a usos industriales y transmiten enfermedades al hombre (1). Muchos de estos casos representaron extremos niveles de pobreza y hambre en toda una población por la pérdida catastrófica de los cultivos y el desabastecimiento de alimento en diferentes regiones del mundo. Se ha calculado que alrededor de un tercio de la producción alimenticia del mundo se perdería si los agricultores no utilizaran algunas medidas para contrarrestar el efecto de las plagas y enfermedades (1). Como consecuencia de estos hechos se desarrolló una serie de productos de origen químico, vendidos como forma de solución al hambre y la pobreza a partir del siglo XIX (1800 - 1920) dando origen a los primeros productos derivados del gas de carbón y a un desarrollo progresivo en cuanto a tecnología y eficiencia (5). Sin embargo, el uso masivo de plaguicidas coincide con la era de la química que ha transformado esta sociedad desde 1950 (2) a través de la denominada "Revolución Verde" incentivada por las gigantescas compañías de agroquímicos como Monsanto, Aventis o Bayer con el argumento de solucionar el hambre y la pobreza que se originaron a partir del XIX. A partir de esto se generó un acelerado incremento en la fabricación de productos agroquímicos y su uso en los campos de cultivo a partir del desarrollo de la industria moderna con un incremento anual del 10\%. En 1981 las ventas en todo el mundo, incluyendo usos no agrícolas, ascendieron a 17500 millones de dólares, de los cuales unos 14,000 millones correspondieron a productos fitosanitarios (1).

Actualmente el uso de agroquímicos ha disminuido en cierto modo, los reportes de casos de cáncer y otras enfermedades han cambiado la perspectiva de mucha gente respecto al consumo de alimentos orgánicos que cada día va creciendo y se va tornando en una alternativa en la producción agrícola en todo el mundo. Sin embargo, aún existen muchos problemas sobre el uso de pesticidas que serán mencionados más adelante.

\section{MECANISMOS $Y$ FORMAS DE CONTAMINACION}

Los agroquímicos son productos utilizados en la agricultura que pueden ser altamente tóxicos y perjudiciales en diferentes aspectos. Los elementos que contienen dentro de su estructura química al ser aplicados continuamente y en forma excesiva a los cultivos, llegan a generar un impacto dentro de 
un ecosistema. Estos elementos sufren una serie de transformaciones posteriores a su manipulación en campo, es así que cerca del $98 \%$ de los insecticidas y el $95 \%$ de los herbicidas utilizados no cumple el objetivo de llegar a la planta y son dispersados a través del viento y el agua. Los componentes tóxicos pueden movilizarse a grandes distancias como residuos volátiles que pasan a la atmósfera y regresan con la lluvia hacia nuevas áreas (6) o a través del suelo (escorrentía, lixiviación o lavado) por medio del agua de riego y lluvia para contaminar fuentes de agua tanto superficial (ríos, lagos y mares) como subterránea.

En la actualidad, uno de los mayores problemas es el uso desmedido y sin control de estos compuestos, tan sólo en 1992 la producción mundial de plaguicidas se estimó en 10 millones de toneladas (6). Sin embargo, existe un desconocimiento de la cantidad y tipos de sustancias que se aplican en los campos; así mismo, el escaso control de los desechos que constantemente se ven expuestos a los factores del medio y que en ocasiones son reutilizados nuevamente (7). También se observa el aumento de las dosis por encima de lo permitido ( 2 - 3 veces más), combinación de productos para maximizar su efectividad y la mala elección de sustancias para controlar plagas y enfermedades que no corresponden, son entre otras cosas parte de una mala práctica agrícola comúnmente realizada en campo.

\section{EFECTOS CONSECUENCIAS. \\ NEGATIVOS}

La agricultura se define como la actividad del hombre sobre la tierra para la extracción de productos de origen vegetal utilizando la energía solar como fuente de energía. Sin embargo, la expansión de nuevas zonas de vida por el hombre a consecuencia del crecimiento de la población mundial ha generado una demanda cada vez mayor de alimentos. Esta actividad trae como consecuencia mayores problemas de carácter irreversible para la naturaleza como: Pérdida en la fertilidad de los suelos, contaminación de los ecosistemas, aparición de nuevas plagas y enfermedades, disminución de la diversidad genética, resistencia genética de organismos perjudiciales, producción de alimentos con residuos tóxicos y eliminación de especies silvestres.

Los plaguicidas dependen mucho de sus características de persistencia para su degradación en el ambiente (8). La pérdida en la fertilidad de los suelos se realiza en elementos indispensables como el fósforo, nitrógeno, potasio y otros (9) que forman parte de la nutrición de las plantas. Estas sustancias reaccionan con los elementos del suelo y forman compuestos no deseados, cambian los valores de $\mathrm{pH}$ y por ende alteran la fauna microbiana producto del desbalance en el medio que los rodea. En el tiempo, los residuos químicos se acumulan y reducen la actividad edáfica y microbiana que sustenta toda actividad agrícola. Por otra parte, la acumulación de residuos puede generar toxicidad en plantas y como parte de este proceso, la fauna silvestre también se ve afectada significativamente por efectos de contaminación de fuentes de agua y por escases de alimentos que mantienen su existencia.

Uvarov en 1964 define que las plagas son el resultado de la ruptura en el balance natural por actividades del hombre. El mismo concepto sustenta la aparición de enfermedades y malezas dentro de un ecosistema agrícola que mediante los métodos de control por erradicación promueven el desarrollo de genes de resistencia como mecanismo natural de defensa para evitar su extinción por el hombre. Cabe mencionar que los métodos de eliminación recomendados por la industria de agroquímicos no son pertinentes al sustituir un problema por otro peor (10).

Las fuentes de agua y el aire sirven de sustento a los ecosistemas naturales y sus 
especies como plantas y animales de la cual dependen en todo el mundo. Desafortunadamente, los sistemas acuáticos son mayormente amenazados por el aporte de sustancias contaminantes por el incremento de actividades antropogénicas en áreas adyacentes que alteran las condiciones naturales, incluyendo al ser humano (7). Los residuos químicos permanecen en el ambiente y pueden llegar hasta los espacios más lejanos del mundo afectando principalmente a peces $y$ animales silvestres. Por ejemplo, los insecticidas organoclorados debido a su estructura molecular resisten la degradación química y bacteriana; y por ser poco solubles al agua se evaporan pasando al aire 0 uniéndose a las partículas del suelo como vapor o polvo. Seguidamente, pueden ser transportados a grandes distancias $y$ nuevamente ser depositadas a través de las lluvias sobre la tierra o aguas superficiales. Cuando llegan al mar, son absorbidas por el plancton que luego servirán de alimento a los peces y estos a sus depredadores, pasando de esta manera a todos los niveles de la cadena trófica. Estas sustancias tienen la propiedad de acumularse en las grasas de los animales y su concentración va aumentando en cada etapa de la cadena para finalmente ser consumidos por el ser humano (11).

La resistencia genética se origina a partir de la presión ambiental antropogénica por selección de individuos dentro de una población de organismos. Los microorganismos patógenos, insectos o malezas logran la supervivencia debido a genes de resistencia que contrarrestan la acción de la materia activa del pesticida, volviéndose un producto sin actividad sobre ese organismo y, por último, formarán descendencia con una población resistente. En general los organismos suceden muy rápidamente y forman poblaciones enormes extendiendo esta característica en pocos años y que pueden ser más destructivos que las generaciones anteriores. Es importante considerar que estos organismos también son seres vivos que desarrollaran mecanismos de supervivencia si el ser humano intenta destruirlos.

\section{REPORTES DE CONTAMINACION DE GRAN IMPACTO}

Los efectos ecológicos de los plaguicidas se encuentran por encima de los métodos de control fitosanitario pudiendo ser uno de los factores más destructivos.

- Se ha referenciado el empobrecimiento de los suelos a causa de los plaguicidas además de la disminución de los microorganismos que cumplen un rol importante en la ecología de las plantas (12).

- La Agencia Europea Para el Medio Ambiente cita un estudio de Galassi en el que vincula estrechamente la toxicidad de los pesticidas en la contaminación del agua del rio Po con impacto directo en el zooplancton Daphnia magna por la escorrentía de los plaguicidas agrícolas (13).

- En los grandes lagos de América del Norte, la bioacumulación y amplificación de los compuestos clorados ha provocado la desaparición de los depredadores superiores, afecciones a la visión y deformidad en especies de aves acuáticas

- El fondo mundial para la naturaleza (WWF) reportó que gran parte de las 190000 toneladas estimadas de plaguicidas agrícolas y no agrícolas descargadas por los países ribereños al mar del norte de Europa son responsables de la mayor tasa de enfermedades, deformidades y tumores en las especies ícticas comerciales y la contaminación del mar del reino unido desde los años setenta (2).

- Según la organización mundial de la salud (OMS, 1993), 4 millones de niños mueren al año como consecuencia de enfermedades diarreicas debidas a infecciones transmitidas por el agua contaminada (14). 
- En un estudio sobre los pozos de agua subterránea en el Ontario occidental agrícola en Canadá, el 35 \% de ellos dieron positivo en las pruebas de plaguicidas (15)

- Además se considera que Brasil se ha convertido en el tercer usuario mundial de sustancias agro tóxicas en el año 1970, superado únicamente por Francia y los Estados Unidos. No obstante, afirma que en este país se analiza menos del $15 \%$ de los ingredientes activos comercializados debido a la falta de metodología, equipos y recursos financieros (16).

- En España se encuentra uno de los lugares más contaminados por pesticidas en el mundo. El río Ebro se ve amenazado por los vertidos ilegales de una fábrica de pesticidas según el estudio realizado por el gobierno de Aragón. Se han reportado desechos por 20000 litros de sustancias tóxicas que representan una catástrofe ambiental (17).

- Según el diario La Página, se reportó el caso de 55 muertos y muchos casos de insuficiencia renal en los habitantes del caserío Loma Linda en el país centro americano de El Salvador como consecuencia de la contaminación de los pozos de agua en la zona. Según los estudios, se mencionan las aspersiones aéreas de plaguicidas en campos de cultivo cercanos a la población por la empresa química agrícola Quimagro (18).

- A finales de los años cuarenta existían pocas especies de insectos resistentes a los pesticidas y en la actualidad se reportan alrededor de 700 especies con esta característica. Al mismo tiempo se han eliminado sus controladores biológicos con frecuencia fundamentales para el equilibrio ecológico. El mismo hecho sucede con las malezas que han creado resistencia a los herbicidas, dando la oportunidad a los fabricantes de agroquímicos de producir plantas transgénicas que no son eliminadas por los herbicidas para aumentar las dosis de aspersión (12).
- Las cifras citadas por muchos organismos mundiales no mencionan la contribución de los pesticidas a múltiples problemas de salud muy frecuentes en las sociedades modernas que van desde los diversos tipos de cáncer, síndrome de fatiga crónica, párkinson y diabetes a pesar de la creciente evidencia científica (12).

\section{ALMIENTOS DE MAYOR EXPOSICION.}

La Enviromental Working Group (2013) ha publicado una lista anual de los productos más contaminados por pesticidas y otros químicos en Estados Unidos. Esta relación se basa en el análisis de 32000 muestras a partir de 48 alimentos de origen vegetal, entre frutas y verduras, realizado por el Departamento de Agricultura de este país. Se determinó que el $65 \%$ de los alimentos resultaron positivos en el contenido de pesticidas principalmente algunos productos agrícolas como manzanas, fresas, uvas, apios, duraznos, espinacas, morrones, nectarinas, pepinos, ajíes y papa considerados entre las más contaminadas. En base a esto se recomienda establecer un estudio basado en los contenidos de residuos tóxicos y los tipos de pesticidas de acuerdo a su grado de toxicidad utilizados por cultivos de manera que se determine las condiciones reales para cada especie agrícola y el informe de los resultados a la población de cada país o región según los derechos de información. Muchos alimentos coinciden respecto al grado de contaminación debido a la presencia de problemas sanitarios que son parte de su proceso productivo, por ejemplo, la alimentación del ganado se basa en el consumo de forraje obtenido a base de agroquímicos y el uso de antibióticos, fármacos y hormonas para evitar enfermedades, son acumulados en el tejido de estos animales que posteriormente serán ofertados como carne y derivados para consumo en los mercados.

En el caso de los productos vegetales, la toxicidad puede incrementarse en los alimentos si los órganos de la planta se encuentran expuestos a la aspersión directa 
durante el control sanitario, aquellos con poco tiempo entre la última aplicación y el momento de la cosecha y los que recibieron productos con alto nivel de toxicidad (etiqueta roja y amarilla). Estos residuos persisten por un tiempo sobre la epidermis de los órganos de la planta si se utilizaron productos de contacto y en el interior de ellos si fueron pesticidas sistémicos, de manera que es difícil saber si un alimento cumplió con las recomendaciones técnicas sobre el uso de agroquímicos. Según estos aspectos, un alimento de apariencia inocua puede representar un gran riesgo para la salud de las personas que lo consumen como es el caso de algunas frutas como las fresas, manzanas, peras, duraznos, uvas; hortalizas como el tomate, papa, además de verduras de hoja como la espinaca y el apio que son expuestos al uso de una gran variedad de productos fitosanitarios y convirtiéndose en alimentos de mayor contaminación, principalmente el tomate.

\section{EFECTOS Y CONSECUENCIAS EN LA SALUD}

Los pesticidas pueden ingresar al cuerpo de una persona de diferentes maneras, pudiendo ser a través de las vías respiratorias, la boca o piel y cuya exposición se dan en forma de partículas de polvo, agua o vapor. En la salud se han realizado investigaciones principalmente a grupos de personas que se dedican a la actividad agrícola con exposición directa a los agroquímicos. En estos casos, los síntomas inmediatos comúnmente conocidos son náuseas, diarrea, ansiedad, dolores abdominales, mareos y confusión como producto de una intoxicación aguda y que pueden ser graves. Por otro lado, en los consumidores (exposición indirecta) los daños pueden darse a largo plazo por ingestión de alimentos tratados, pero con condiciones de inocuidad aparentes. Cabe resaltar, que estos residuos químicos debido a su propiedad de persistencia se mantienen y acumulan en el cuerpo a pesar de ingerir dosis insignificantes pero que en un futuro puede representar un riesgo importante.
Finalmente, se ha demostrado las consecuencias del uso inadecuado de los productos químicos en la producción de alimentos respecto a la salud de todo organismo, tanto animales como seres humanos, siendo asociados problemas como el cáncer, deformaciones congénitas, alteraciones hormonales, deficiencias en el sistema reproductivo, daños celulares, problemas respiratorios, trastornos de memoria, enfermedades de la piel, depresión, abortos y enfermedades neurológicas. Adicionalmente, se ha determinado algunos grupos de mayor riesgo dentro de una población, siendo principalmente los fetos, bebés, niños y adolescentes por encontrarse en proceso de crecimiento y desarrollo además de las mujeres embarazadas, lactantes o mujeres en edad fértil (19).

\section{MEDIDAS PREVENTIVAS}

Al respecto, se indica que es necesario hacer una reglamentación en el uso y aplicación de estos compuestos, sobre todo en los registros y programas de vigilancia sobre la contaminación ambiental e intoxicación. También se considera lixiviados de los campos agrícolas que pueden llegar a los mantos freáticos producto de la aplicación en exceso de los agroquímicos $(12,20)$.

Los Organismos Internacionales, como la FAO (Organización para la Agricultura y Alimentación) y la Organización Mundial de la Salud (OMS), han establecido los niveles máximos admisibles respecto a la ingestión de plaguicidas normalmente utilizados en distintos países, siendo las autoridades nacionales las encargadas de establecer una legislación apropiada y vigilar cuidadosamente los residuos de los plaguicidas mediante controles analíticos adecuados (1). Según lo mencionado, el uso racional de los plaguicidas puede concretarse mediante una legislación de productos químicos a través de la implementación de controles y análisis con extensión de la información sobre pesticidas. Al mismo tiempo, se debe adoptar las medidas 
adecuadas para garantizar al usuario que los productos sean eficaces y seguros (1).

Finalmente, las medidas de prevención para contrarrestar el impacto ambiental y de salud deben complementarse con una serie de acciones que incluyen el mejoramiento de los sistemas de prevención durante el control de enfermedades, reducción en el uso de plaguicidas mediante prácticas relacionadas a la agricultura orgánica, prohibición de productos con ingredientes activos altamente tóxicos y que representan un peligro para el ambiente y la salud, formulación de agroquímicos en forma pertinente respecto a las dosis, mezclas o elección de productos y finalmente el manejo adecuado de residuos tóxicos utilizados.

\section{Correspondencia:}

Ing. Marco Antonio Ramírez Campos

Correo electrónico: Marco.rc@hotmail.com

\section{REFERENCIAS BIBLIOGRAFICAS}

1. Sánchez M.J., Sánchez M. Los plaguicidas, adsorción y evolución en el suelo. Instituto de recursos naturales y agrobiología. (Internet) Boletín. 1era ed. Salamanca, España; 1984. ( Citado el 12 de Octubre 2017) Disponible en: digital.csic.es/handle/10261/12919.

2. Ongley E. D. Lucha contra la contaminación agrícola de los recursos hídricos. Estudio FAO riego y drenaje - 55. GEMS/ Water Collaborating Center. Burlington, (Internet). Canadá; 1997. 115 p. (Citado el 12 de Octubre 2017) Disponible en: www.fao.org/docrep/W2598S/w2598s00.htm

3. Galán E, Gómez J. L, Bellinfante N, Aparicio P. Contaminación de suelos por compuestos orgánicos. Informe final. (Internet). Sevilla, España; 2003. 185 p. (Citado el 12 de Octubre 2017) Disponible en:

www.juntadeandalucia.es/medioambiente $>$ si te>portalweb.

4. Agrios G. N. Fitopatología. Universidad de Massachusetts. Massachusetts. 2da ed .Estados Unidos; 2007. 158p.
5. Stephenson G. A, Solomon K. R. Pesticides and environment. Department of environmental Biology. University of Guelph. Canadá; 1993.

6. López J. A, Martínez C, Moreno L, Navarrete P. Las aguas subterráneas y los plaguicidas. Instituto Geológico y minero de España; 1992. 149 p. ( Citado el 18 de Noviembre 2017) Disponible en: aguas.igme.es/igme/publica/libro28/lib28.ht $\underline{\mathrm{m}}$

7. García, C. Rodríguez G. D. 2012. Problemática y riesgo ambiental en Sinaloa. (Internet). Rev Sociedad, Cultura y Desarrollo Sustentable. Universidad Autónoma Indígena de México. Sinaloa. México.2012; 8(3): 1-10. (Citado el 18 de Noviembre 2017) Disponible en: www.redalyc.org/pdf

8. México, Secretaria de Medio Ambiente y Recursos Naturales (SEMARNAT). Indicadores básicos del desempeño ambiental en México (Internet). México, D.F; 2005. 337p (Citado el 11 de Noviembre 2017). Disponible en: apps1.semarnat.gob.mx/dgeia/indicadores 1 4/conjuntob/00 conjunto/ introducción.htm.

9. Orozco M. A. Fomento de la agricultura sostenible mediante el establecimiento de un sistema de garantías de calidad en los procesos productivos y de comunicación a los consumidores. Aplicación a la agricultura mexicana (Internet). Tesis Doctoral. Universidad Politécnica de Cataluña. Barcelona, España; 2006. 371 p. (Citado el 18 de Noviembre 2017) Disponible en: $\underline{\text { www.tdx.cat }>\text { bitstream }>\text { handle }}$

10. Uvarov B. P. Problems of insect ecology in developing countries. Anti-Locust Research Centre. British Ecological Society. London, Ingland (Internet). Journal of Applied Ecology.1964; 1(1): 159-168. ( Citado el 18 de Noviembre 2017 ) Disponible en: www.jstor.org/stable/2401595. 
11. Anguiano O. L, Souza M. S, Ferrari A, Soleño J, Pechen A.M, Montagna C. M. Los plaguicidas y sus efectos sobre el medio ambiente. facultad de ingeniería argentina. (Internet).Argentina; 2011. (Citado el 18 de Noviembre 2017 ) Disponible en: www.lareserva.com/home/plaguicidas pesti cidas efectos medio ambiente

12. FAO. Código Internacional de conducta para la distribución y utilización de plaguicidas. Organización de las Naciones Unidas para la agricultura y la alimentación (Internet) Roma; 2006. 40 P. (Citado el 6 de Diciembre 2017 ) Disponible

en: www.fao.org/docrep/006/Y4544S/Y4544S00 $\underline{\text { htm }}$

13. EEA. European river and lakes: assesment and their enviromental state. Agencia europea del medio ambiente (Internet). Monograph No 1. Copenhague, Dinamarca; 1994.(Citado el 6 de Diciembre 2017 ) Disponible en:

www.eea.europa.eu/publications/87-90198$\underline{01-8}$

14. Organización mundial para la salud. Guías para la calidad del agua potable. Recomendaciones. $2^{\text {da }}$ ed. Vol 1 (Internet). OMS, Ginebra; 1993. (Citado el 6 de Diciembre 2017). Disponible en: www.who.int/water sanitation health/dwq/gdwq3rev/es/

15. Lampman M. Susceptibility of groundwater to pesticide and nitrate contamination and predisposed áreas of southwestern Ontario. Water Quality Research Journal of Canada.1995; 30(3):443-468.

16. Andreoli, C V. The influence of agriculture on water quality. En: Prevention of Water Pullution by Agricultura Related Activites. Wáter report 1. FAO (Internet). Roma; 1993. p 53 - 65. (Citado el 6 de Diciembre 2017 ) Disponible en:

www.extension.usu.edu>agriculturewq.
17. Ansede M. Uno de los lugares más contaminados por pesticidas en el mundo está en España (Internet). Diario Materia. Madrid, España; 2013. (Citado el 6 de Diciembre 2017 ) Disponible en: www.esmateria.com/2013/02/06/Uno_de _ los lugares más contaminados por pestici das en el mundo está en Espana/

18. Menéndez $\mathbf{S}$. Contaminación por pesticidas provoca graves enfermedades en San Luis talpa (Internet). Diario la Página. La Paz, El Salvador; Publicado el 6 de Junio 2013. (Citado el 10 de Diciembre 2017) Disponible en: www.lapagina.com.sv/nacionales/82417/ Contaminación por pesticidas provoca gra ves enfermedades en San Luis Talpa.

19. CENTRO DE ECOGENÉTICA Y SALUD AMBIENTAL. 2012. Riesgos a la salud por pesticidas en los alimentos. Universidad de Washington (Internet). Boletín; 2012. 2p. (Citado el 10 de Diciembre 2017 ) Disponible en:

depts.washington.edu>FF Pesticides SP

20. Garrido T, Costa C, Fraile J, Orejudo E, Niñerota J, Ginebreda A, et al. Análisis de la presencia de plaguicidas en diversos acuíferos de Cataluña. Jornadas sobre la contaminación de las aguas subterráneas: un problema pendiente (Internet). Valencia, España; 1998. 7 p. (Citado el 10 de Diciembre 2017) Disponible en: www.researchgate.net/publication/26782776 0 análisis de la presencia de plaguicidas en diversos acuíferos de cataluña.

\section{Recibido: 02/08/2018 \\ Aprobado para Publicación: 30/10/2018}

